\title{
Réacteurs membranaires à enzymes immobilisées
}

\author{
par \\ Annie MARC*, G. DUC*, J. M. ENGASSER* \\ et Q. T. NGUYEN**
}

\section{Ré s u m é}

La mise en œuvre de procédés enzymatiques et microbiologiques dans des modules d'ultrafiltration présente de nombreux avantages en biotechnologie. Une approche consiste à immobiliser l'enzyme à l'intérieur de membranes asymétriques par perméation de la solution enzymatique à travers la membrane. Nous avons adapté cette méthode à l'immobilisation de la glucoamylase dans des modules à fibres creuses, utilisés couramment comme hémodialyseurs, et prétraités par une réticulation entre du glutaraldéhyde et de l'albumine. Dans ce cas, l'enzyme ne subit aucun relargage à travers la membrane et les performances du réacteur restent stables pendant plusieurs mois. L'évolution de l'activité enzymatique initiale du réacteur a été étudiée en fonction, d'une part de la quantité d'enzyme immobilisée, et d'autre part, de la concentration du substrat. L'ensemble des essais a été réalisé avec des solutions de substrat concentrées et à des débits élevés. Les performances catalytiques d'un tel réacteur sont tout à fait comparables à celles obtenues avec d'autres types de réacteurs à enzymes immobilisées. Il présente en plus de nombreux avantages dont les principaux sont les suivants : immobilisation rapide, régénération aisée, grande surface volumique, grandes capacités de transfert externes, micro-environnement de l'enzyme peu influencé, peu de besoins énergétiques.

Mots clés : Fibres creuses - Glucoamylase - Maltose - Amidon - Réacteur enzymatique - Diffusion.

* Laboratoire des Sciences du génie chimique, C.N.R.S.-E.N.S.I.C., 1, rue de Grandville - 54042 Nancy cedex.

** Laboratoire de Chimie physique macromoléculaire, E.N.S.I.C., 1, rue de Grandville - 54042 Nancy cedex. 


\section{S u m m a r y}

Ultrafiltration modules, used for enzymatic an microbiological processes, present many attractive features in biotechnology. An alternative is to immobilize the enzymes into asymmetric membranes by permeation of the enzyme solution across the membrane. We have adapted this method at glucoamylase immobilization in hollow fiber modules, generally used as haemodialyser, and treated by glutaraldehyde and albumin. In this case, there is no enzyme leakage across the membrane. Reactor performances are stables during several months. Dependence of initial enzymatic activity was studied first on the immobilized enzyme amount and secondly on the substrate concentration. All experiments were made with high substrate concentrations and high flow rates. Catalytic performances of this reactor are comparable with other immobilized enzyme reactors. Its main advantages are a fast immobilization, an easy regeneration, a large ratio of surface area to volume, an high external transport capacity, a low alteration of enzyme microenvironment and low energy requirements.

Key words: Hollow fiber - Glucoamylase - Maltose - Starch - Enzyme reactor - Diffusion.

\section{INTRODUCTION}

Des procédés biotechnologiques mettant en œuvre des catalyseurs biologiques sont couramment utilisés dans l'industrie agro-alimentaire, pharmaceutique ou biochimique. L'immobilisation des biocatalyseurs présente des avantages tels que la réutilisation du catalyseur, une productivité plus grande par unité de volume de réacteur, la possibilité de procédés en continu et l'obtention de produits plus purs. Une façon d'immobiliser une enzyme à l'intérieur d'un réacteur tout en la gardant en solution est de la compartimenter à l'aide de membranes d'ultrafiltration, par exemple dans des cartouches à fibres creuses. Cette utilisation de modules d'ultrafiltration a été amorcée vers 1970 (Rony, 1971). De nombreux travaux de recherches ont été réalisés depuis (Van Dongen et Cooney, 1977; Lewis et Middleman, 1974 ; Chambers et coll., 1976 ; Engasser et coll., 1980). D'autres études portent sur la description théorique de ce type de réacteur en introduisant les effets diffusionnels (Waterland et coll., 1974; Kim et Cooney, 1976; Kataoka et coll., 1980). Cependant, depuis 1980, plusieurs auteurs mentionnent des problèmes de fonctionnement comme, par exemple, l'adsorption de certains substrats (Kohlwey et Cheryan, 1981), et le relargage de l'enzyme (Silman et coll., 1980; Deeslie et Cheryan, 1982). 
C'est pourquoi, nous nous sommes attachés, dans ce travail, à la mise au point d'un réacteur à fibres creuses possédant une forte activité catalytique ainsi qu'une bonne stabilité. La réaction étudiée, d'une grande importance industrielle, est l'hydrolyse de l'amidon par la glucoamylase. Les études ont été réalisées en se rapprochant le plus possible des conditions industrielles (enzyme non purifiée, substrat industriel, concentrations élevées).

\section{MATERIEL ET METHODES}

Pour cette étude, notre choix s'est porté sur l'hémolfiltre capillaire Gambro FH202, couramment utilisé comme hémodialyseur. Ce réacteur répond en effet correctement aux principaux critères de choix fixés (grande surface membranaire, débits élevés possibles, bonne résistance mécanique, encombrement réduit). Il est constitué de 6500 fibres creuses, de $25 \mathrm{~cm}$ de long et de $200 \mu \mathrm{m}$ de diamètre interne, représentant une surface efficace de $1,2 \mathrm{~m}^{2}$. La membrane asymétrique des fibres comporte une fine membrane sélective interne et un support macroporeux externe de $20 \mu \mathrm{m}$ d'épaisseur. Son seuil de rétention moyen se situe aux environs de 30000 .

Afin d'améliorer la rétention de la glucoamylase dont le poids moléculaire moyen est de 60000 , un prétraitement par du glutaraldéhyde et de l'albumine a été réalisé à l'intérieur du support macroporeux. Celui-ci a pour objectif de réduire les pores de la membrane sélective et abaisser ainsi son seuil de coupure.

L'immobilisation de la glucoamylase OPTIDEX (Miles) à l'intérieur du support macroporeux a été conduite par perméation de la solution enzymatique à travers la membrane de l'extérieur vers l'intérieur de la fibre.

Lors du fonctionnement du réacteur, le substrat est pompé, à un débit fixé, à travers le volume interne des fibres avant d'être recyclé dans un réservoir agité. L'ensemble de l'installation est thermostaté à $40^{\circ} \mathrm{C}$ et les prélèvements au cours du temps sont réalisés dans le réservoir. Le volume externe de la cartouche est vide et fermé. La réaction s'effectue à $\mathrm{pH}$ 4,6.

Deux substrats ont été testés : le maltose (Merck) et une maltodextrine (Roquettes Frères) résultant d'une préhydrolyse enzymatique d'amidon de maïs. Les concentrations utilisées varient de 20 à $400 \mathrm{~g} / \mathrm{l}$. Les produits d'hydrolyse ont été analysés par HPLC sur une colonne $\mu$-BONDAPAk Carbohydrates (Waters Associate) avec un mélange eau-acétonitrile. L'activité initiale du réacteur est définie comme la quantité de glucose (en grammes) produite par minute. 


\section{RESULTATS ET DISCUSSION}

\section{Efficacité du gel protéique sur la rétention enzymatique}

L'immobilisation de l'enzyme s'effectue par perméation de la solution enzymatique de l'extérieur vers l'intérieur des fibres. Afin d'améliorer la rétention enzymatique, nous avons testé un prétraitement du réacteur conduisant à la mise en place d'un gel protéique à l'intérieur du support macroporeux. La figure 1 donne une représentation schématique d'une fibre prétraitée vue en coupe.

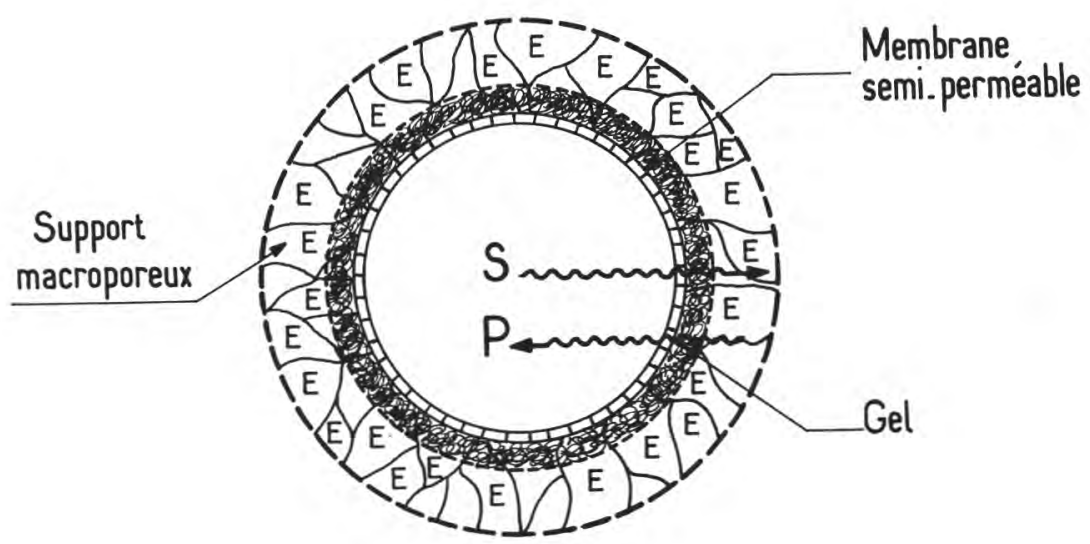

fig. 1

Représentation schématique d'une fibre à membrane asymétrique vue en coupe après dépôt du film protéique.

Schematical cross-section of an asymetric hollow-fiber with proteinic gel.

La figure 2 compare les résultats obtenus lors de l'immobilisation de l'enzyme sans ou avec dépôt du film protéique. On observe que, sans aucun prétraitement, près de $67 \%$ de la quantité d'enzyme initialement en solution traverse la membrane vers l'intérieur des fibres. Par ailleurs, si l'on perfuse le réacteur à l'intérieur des fibres avec une solution tamponnée, des tests d'activité enzymatique de celle-ci à différents instants montrent un relargage de l'enzyme qui atteint $5 \%$ de la quantité initiale en $24 \mathrm{~h}$.

Ce résultat peut s'expliquer par le fait que le seuil de coupure de la membrane s'étend de $10^{4}$ à $10^{5}$. Or, la glucoamylase est constituée de trois isoenzymes dont les poids moléculaires se situent entre 38000 et 76000 . Certaines molécules enzymatiques sont donc aptes 
fig. 2

Efficacité du gel protéique sur la rétention enzymatique : sans $(\rightarrow$ ) et avec (-. - - $)$ prétraitement.

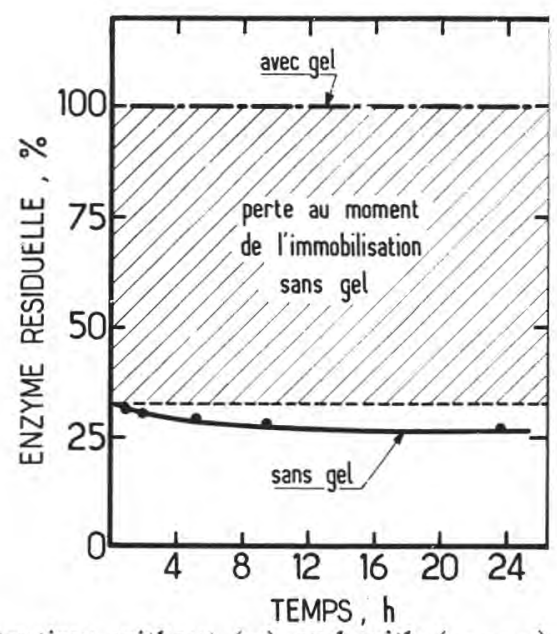

Proteinic gel efficiency on enzymatic retention: without ( $\rightarrow$ ) and with (-. - treatment.

à traverser la membrane. D'autres études montrent également la présence d'un relargage enzymatique, moins marqué mais non négligeable, même avec des membranes de seuil de coupure beaucoup plus faible de 5000 et 10000 . Cette perte d'enzyme peut être provoquée par une irrégularité des pores du fait de la méthode statistique de fabrication de la membrane ou par d'autres phénomènes difficiles à contrôler (confinement des molécules, effet de pompe à membrane, mauvais empotage des fibres, etc.).

Par contre, le prétraitement du réacteur permet une rétention complète de l'enzyme au moment de son immobilisation. Lors du fonctionnement du réacteur, les tests de relargage se sont révélés négatifs. On dispose donc d'un réacteur sûr empêchant toute contamination enzymatique des solutions à traiter.

\section{Activité du réacteur à fibres creuses - Rendement d'immobilisation}

Dans cette partie, nous avons déterminé l'activité du réacteur en fonction de la quantité d'enzyme immobilisée. Ce travail a été réalisé à deux concentrations de maltose : $20 \mathrm{~g} / 1$ et $400 \mathrm{~g} / 1$. Pour chaque quantité d'enzyme immobilisée on calcule un rendement d'immobilisation défini comme le rapport de l'activité initiale du réacteur (en g. $\mathrm{min}^{-1}$ ) à l'activité de la même quantité d'enzyme soluble (en g.min ${ }^{-1}$ ).

Avec la plus faible concentration de maltose, l'activité maximale, égale à $0,38 \mathrm{~g} \cdot \mathrm{min}^{-1}$, est atteinte pour $30 \mathrm{ml}$ d'enzyme avec un rendement de l'ordre de $8 \%$ (fig. 3a). Par contre, à $400 \mathrm{~g} / 1$ de maltose, l'activité maximale du réacteur égale à $11,5 \mathrm{~g} \cdot \mathrm{min}^{-1}$, est atteinte pour 

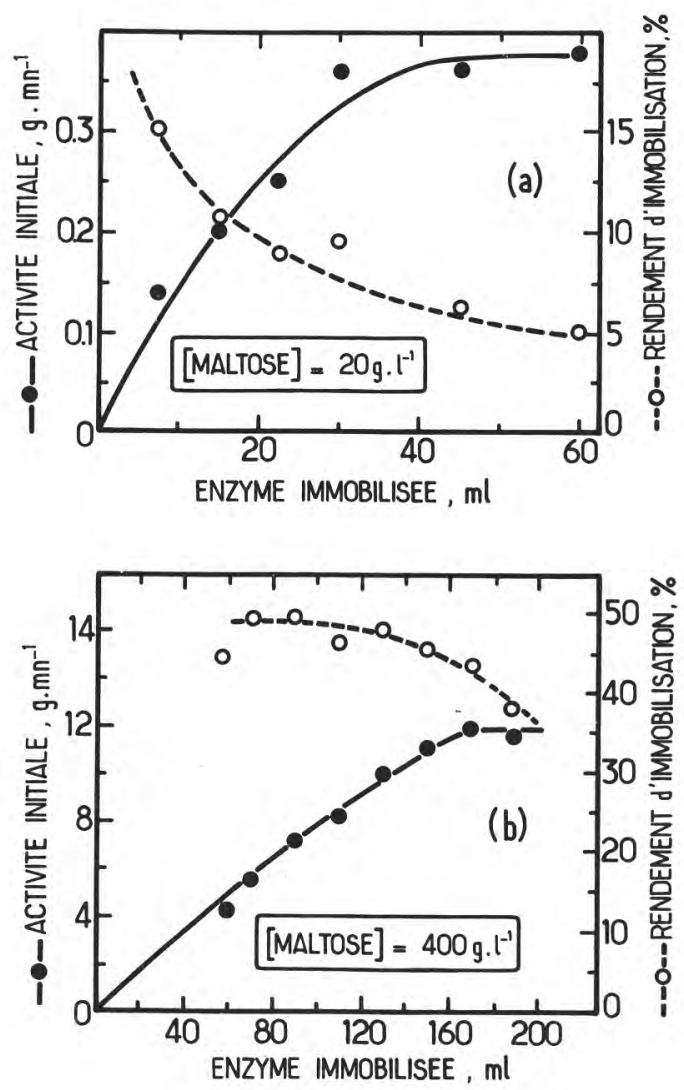

fig. 3

Evolution de l'activité initiale du réacteur et du rendement d'immobilisation en fonction de la quantité d'enzyme immobilisée ([maltose $]=20 \mathrm{~g} / \mathrm{l}$ (a) et [maltose $]=400 \mathrm{~g} / \mathrm{l}(\mathrm{lb}))$.

Dependance of reactor activity and immobilization yield on immobilized enzyme amount ([maltose $]=20 \mathrm{~g} / \mathrm{l}(\mathrm{a})$ and $[$ maltose $]=400 \mathrm{~g} / \mathrm{l}(\mathrm{b}))$.

$170 \mathrm{ml}$ d'enzyme, tandis que le rendement se situe aux environs de $40 \%$ (fig. $3 b$ ).

Le rendement maximal observé, même aux faibles concentrations enzymatiques, ne dépasse pas $50 \%$. Cette perte d'activité de l'enzyme immobilisée par rapport à l'enzyme soluble peut être due à divers phénomènes : dénaturation enzymatique, empêchement stérique, etc. Par contre, alors que l'activité de l'enzyme soluble augmente de façon linéaire avec la concentration enzymatique, on observe, dans le cas de la glucoamylase immobilisée, la présence d'un palier d'acti- 
vité aux fortes concentrations enzymatiques. Ce résultat est le fait de la présence de limitations diffusionnelles et est illustré par les schémas de la figure 4. La figure 4a représente l'évolution du profil des concentrations du substrat au fur et à mesure que l'on augmente la quantité d'enzyme à l'intérieur du support poreux.

La réaction s'effectue en trois étapes : diffusion externe du substrat dans le milieu vers la membrane, diffusion à l'intérieur

(a)
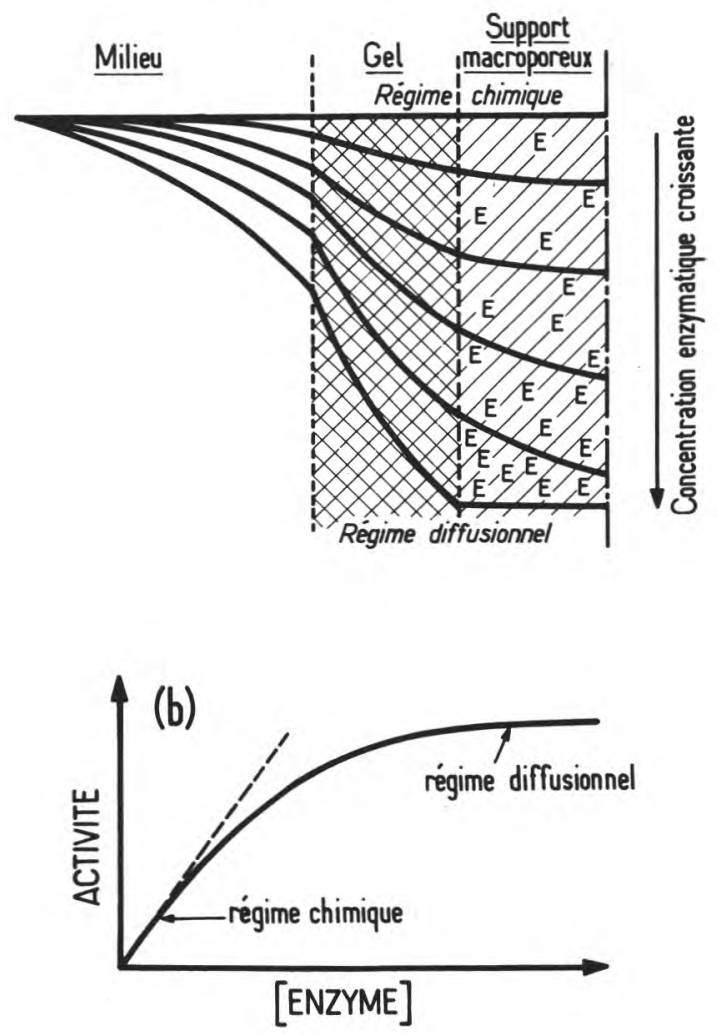

fig. 4

Représentation schématique de l'évolution du profil des concentrations de substrat en fonction de la concentration enzymatique (a) et de l'influence des limitations diffusionnelles sur l'activité en fonction de la concentration enzymatique (b).

(a) Schematical dependence of substrate concentrations on enzyme concentrations.

(b) Schematical dependence of reactor activity on enzyme concentration with diffusionnal limitations. 
de la couche de gel, et diffusion et hydrolyse enzymatique simultanée à l'intérieur du support macroporeux. L'étape limitant la vitesse de la réaction est celle caractérisée par la plus faible vitesse maximale. A une certaine concentration élevée d'enzyme, le substrat est hydrolysé dès qu'il atteint le support macroporeux et sa concentration s'annule. La vitesse de la réaction n'est donc pas limitée par la réaction chimique qui est très rapide ni par la diffusion à l'intérieur des pores. Elle devient égale à la vitesse maximale permise par le transfert externe et à l'intérieur du gel. On dit alors que le réacteur se trouve en régime limite diffusionnel (fig. 4b). A l'opposé, aux faibles concentrations d'enzyme le réacteur est en régime limite chimique.

On peut calculer la vitesse maximale de transfert externe vers la membrane aux deux débits utilisés : $100 \mathrm{ml} / \mathrm{min}$ et $500 \mathrm{ml} / \mathrm{min}$. Elle vaut 9,6 g.min ${ }^{-1}$ pour une concentration de substrat de $20 \mathrm{~g} / \mathrm{l}$ et $247 \mathrm{~g} \cdot \mathrm{min}^{-1}$ pour une concentration de $400 \mathrm{~g} / \mathrm{l}$. Les paliers d'activités maximales observés se situent à des valeurs nettement inférieures à celles permises par le transfert externe. Ce dernier n'est donc pas le phénomène limitant. On peut supposer qu'il existe une forte barrière diffusionnelle due à la présence de la couche de gel qui détermine toute la cinétique du réacteur.

La deuxième observation de cette étude est la comparaison des activités maximales obtenues aux deux concentrations de substrat. La vitesse maximale de diffusion à travers le gel augmente avec la concentration de substrat, le gradient de concentration étant plus élevé. Ceci explique la plus forte activité maximale observée avec la concentration de $400 \mathrm{~g} / 1$.

\section{Stabilité du réacteur au stockage}

Cette étude a été réalisée avec un réacteur non saturé en enzyme $(7,5 \mathrm{ml})$ sur des solutions de maltose de $20 \mathrm{~g} / \mathrm{l}$, donc, très certainement, dans la zone d'activités contrôlées par la réaction chimique et non par la diffusion. Entre chaque mesure d'activité le réacteur est stocké, à $4^{\circ} \mathrm{C}$, dans une solution d'acide de sodium à $0,02 \%$ et $\mathrm{pH} 4,6$. La stabilité a été mesurée pendant 260 jours soit presque 9 mois. La figure 5 représente les résultats obtenus. Ils montrent une très bonne stabilité de l'activité catalytique du réacteur qui ne diminue que de $20 \%$. Nous avons donc obtenu ainsi un réacteur enzymatique, non seulement sans relargage d'enzyme, mais dont l'activité possède une remarquable stabilité.

\section{Influence de la concentration de substrat sur l'activité du réacteur}

Dans un dernier temps, nous avons mesuré l'activité du réacteur en fonction de différentes concentrations initiales de substrat. La figure 6 représente les résultats obtenus avec le maltose alors que 
fig. 5

Evolution de l'activité du réacteur

au cours du stockage.

Dependence of reactor activity on storage.

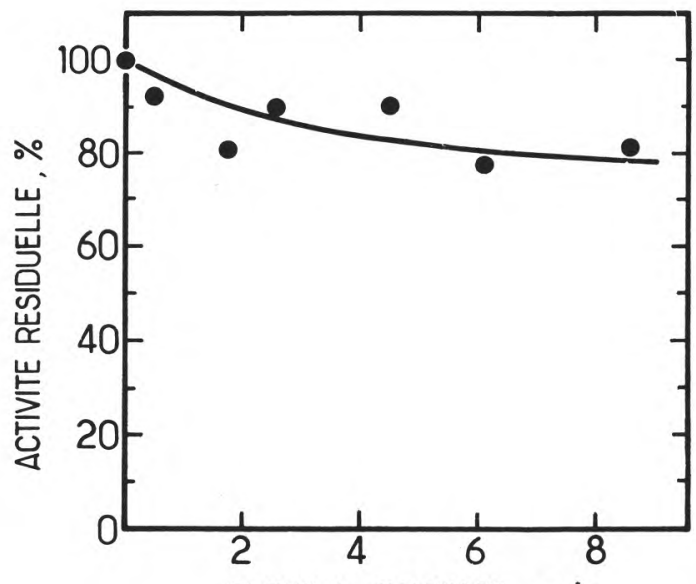

DUREE du STOCKAGE, mois

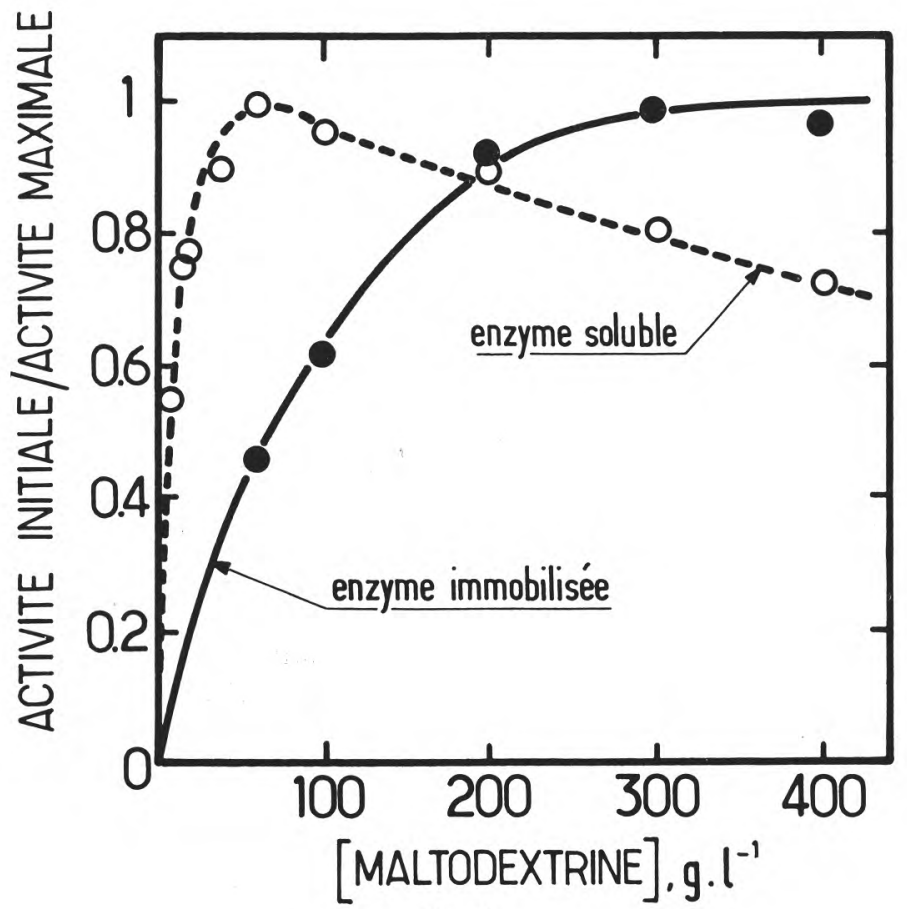

fig. 6

Comparaison des activités de l'enzyme soluble et immobilisée en fonction de la concentration de maltose.

Dependence of soluble and immobilized enzyme activity on maltose concentration. 
fig. 7

Comparaison des activités de l'enzyme soluble et immobilisée en fonction de la concentration de maltodextrine.

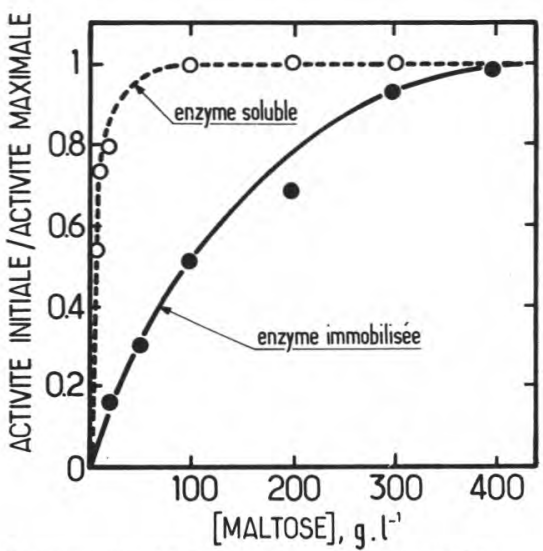

Dependence of soluble and immobilized enzyme activity on maltodextrin concentration.

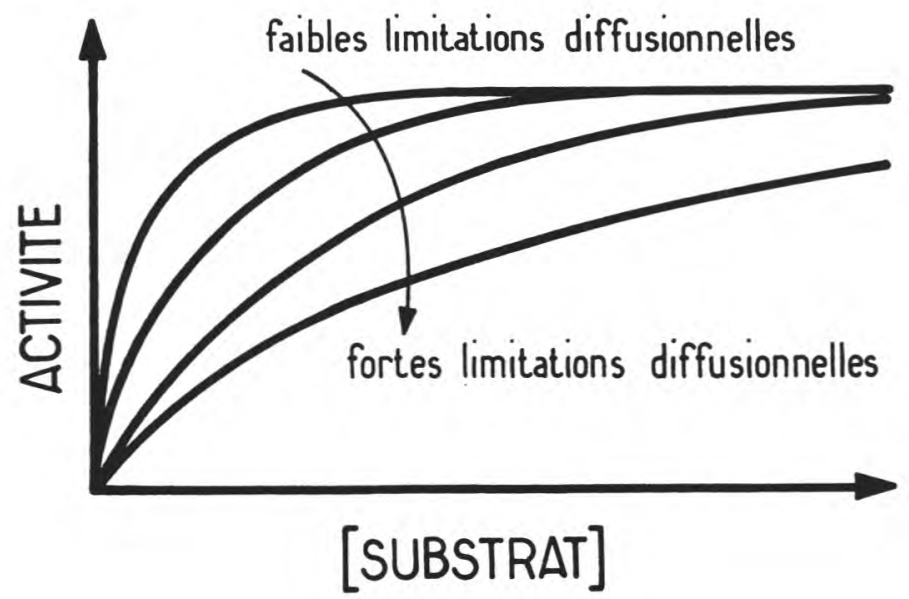

fig. 8

Représentation schématique de l'influence des limitations diffusionnelles sur l'activité en fonction de la concentration de substrat.

Schematical dependence of reactor activity on substrate concentration with diffusionnal limitations.

la figure 7 donne les résultats obtenus avec la maltodextrine. Pour faciliter la comparaison avec l'activité de l'enzyme soluble nous avons reporté le rapport de l'activité mesurée sur l'activité maximale. Ceci permet de faire abstraction des phénomènes intervenant lors de l'immobilisation, tels que la dénaturation des molécules protéiques ou des empêchements stériques pour ne garder que l'influence des limitations diffusionnelles. On note, avec le maltose, une forte aug- 
mentation de la concentration de demi-vitesse maximale lorsque l'enzyme est immobilisée. L'allure des courbes se rapproche de celles de la figure 8 où est schématisée l'influence des limitations diffusionnelles sur l'activité en fonction de la concentration de substrat. Ces résultats confirment bien la présence d'une barrière diffusionnelle. Les mêmes conclusions peuvent être tirées des expériences réalisées avec la maltodextrine. De plus, alors qu'avec l'enzyme soluble on note une inhibition aux fortes concentrations de dextrines, celle-ci reste inapparente avec le réacteur à glucoamylase immobilisée.

\section{CONCLUSION}

La mise en œuvre d'un hémofiltre à fibres creuses asymétriques pour l'immobilisation d'enzymes apparaît, après l'exposé de ces travaux, comme particulièrement intéressante. Les activités catalytiques maximales obtenues avec la glucoamylase, dans des conditions proches des conditions industrielles, sont tout à fait comparables à celles obtenues avec d'autres méthodes d'immobilisation (tab. 1). Par ailleurs, ce type de réacteur présente d'autres caractéristiques avantageuses. L'immobilisation de l'enzyme est particulièrement simple et efficace, de même que sa régénération. L'absence de relargage d'enzyme est bien adaptée pour des procédés où une contamination enzymatique est strictement interdite. La stabilité de l'activité catalytique reste un paramètre important. La grande surface membranaire du module $\left(4000 \mathrm{~m}^{2} / \mathrm{m}^{3}\right)$ permet une grande productivité par unité de volume de réacteur et implique des volumes d'installation réduits. Dans un tel système polytubulaire, les pertes de charges sont inférieures à celles rencontrées dans des réacteurs à lit fixe et limitent donc l'énergie nécessaire pour l'écoulement du milieu.

L'activité de ce réacteur présente une réduction par rapport à celle obtenue avec l'enzyme soluble provoquée par divers phénomènes (dénaturation enzymatique, empêchements stériques, etc.). Aux fortes concentrations de substrat, le rendement d'immobilisation est de l'ordre de $40 \%$, ce qui est supérieur, en règle générale, aux autres méthodes d'immobilisation. On a pu noter de fortes limitations diffusionnelles provoquées par la présence du gel de prétraitement, principal facteur limitant l'activité du réacteur. Le calcul de ce type de réacteur se trouve ainsi compliqué du fait de la présence de ces limitations diffusionnelles. Mais il peut être maîtrisé grâce à l'utilisation des théories du génie biochimique.

L'application de ce réacteur enzymatique pour des productions en petites quantités de substances à haute valeur ajoutée est tout à fait envisageable. De plus, ce type de module d'ultrafiltration apparaît bien adapté à la culture de micro-organismes et même de cellules animales, en particulier lorsque les produits du métabolisme, toxiques pour la croissance, doivent être éliminés. 
TABLEAU 1 - TABLE 1

Comparaison des activités catalytiques obtenues avec divers types de réacteurs à glucoamylase immobilisée Comparison between different glucoamylase immobilized reactors activities

\begin{tabular}{|c|c|c|c|c|c|c|c|c|}
\hline & SUPPORT & $\begin{array}{l}\text { Présente } \\
\text { étude }\end{array}$ & $\begin{array}{l}\text { Fibres } \\
\text { creuses }\end{array}$ & $\begin{array}{c}\text { Fibres } \\
\text { cellulosiques }\end{array}$ & $\begin{array}{c}\text { Céramique } \\
\text { poreuse }\end{array}$ & $\begin{array}{l}\text { Alumine } \\
\text { poreuse }\end{array}$ & $\begin{array}{l}\text { Membrane } \\
\text { de collagène }\end{array}$ & $\begin{array}{l}\text { Carbone } \\
\text { activé }\end{array}$ \\
\hline \multirow[t]{2}{*}{ 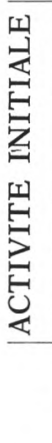 } & $\begin{array}{l}\text { mole.mn-1 } \\
\mathrm{cm}^{-3} \text { réacteur } \\
\text { mole.mn-1 } \\
\mathrm{cm}^{-2} \text { support } \\
\text { mole.mn }{ }^{-1} \\
\mathrm{~g}^{-1} \text { support }\end{array}$ & $\begin{array}{l}204.10^{-6} \\
5,1.10^{-6} \\
3,05.10^{-3}\end{array}$ & $0,7.10^{-6}$ & $57.10^{-6}$ & $7.10^{-6}$ & $0,15 \cdot 10^{-3}$ & $4,6.10^{-6}$ & $0,12 \cdot 10^{-3}$ \\
\hline & REFERENCE & - & $\begin{array}{l}\text { Engasser } \\
\text { et } \text { coll., } 1980\end{array}$ & $\begin{array}{l}\text { Corno } \\
\text { et } \text { coll., } 1972\end{array}$ & $\begin{array}{l}\text { Weetall } \\
\text { et } \text { coll., } 1976\end{array}$ & $\begin{array}{c}\text { Allen } \\
\text { et coll., } 1979\end{array}$ & $\begin{array}{c}\text { Brillouet } \\
\text { et } \text { coll., } 1977\end{array}$ & $\begin{array}{c}\text { Cho et } \\
\text { Bailey, } 1978\end{array}$ \\
\hline
\end{tabular}




\section{Bibliographie}

Allen (B. R.), Charles (M.), Coughlin (R. W.) (1979). - Fluidized-bed immobilized enzyme reactor for the hydrolysis of cornstarch to glucose. Biotechnology and Bioengineering, 21, 689-706.

Brillouet (J. M.), Coulet (P. R.), Gautheron (D. C.) (1977). - Chemically activated collagen for amyloglucosidase attachment. Use in a helicoidal reactor. Biotechnology and Bioengineering, 19, 125-142.

Chambers (R. P.), Cohen (W.), Baricos (W. H.) (1976). - Physical immobilization of enzymes by hollow-fiber membranes. In " Methods in enzymology, immobilized enzymes ", edited by Mosbach K., Academic Press, New York.

CHo (Y. K.), BAILEY (J. E.) (1978). - Immobilization of enzymes on activated carbon: Properties of immobilized glucoamylase, glucose oxidase, and gluconolactone. Biotechnology and Bioengineering, 20, 1651-1665.

Corno (C.), Galli (G.), Morisi (F.), Bettonte (M.), Stopponi (A.) (1972). - Glucoamylase entrapped into cellulosic fibres. Die Stärke, 24, 420-424.

DeEsLIE (W. D.), Cheryan (M.) (1982). - A CSTR-Hollow-Fiber system for continuous hydrolysis of proteins. Factors affecting long-term stability of the reactor. Biotechnology and Bioengineering, 24, 69-82.

Engasser (J. M.), CAumon (J.), MARc (A.) (1980), - Hollow fiber enzyme reactors for sugar and starch processing. In « Food process engineering: Enzyme engineering in food processing ", edited by Linko P. and Larinkari J., Applied Science Publishers LTD, London.

Kataoka (H.), Saigusa (T.), Mukataka (S.), Takahashi (J.) (1980). - Effects of axial dispersion and mass transfer resistances on conversion in a hollow fiber enzyme reactor. J. Ferment. Technol., 58, 5, 431-437.

KiM (S. S.), CoONEY (D. O.) (1976). - An improved theoretical model for hollow fiber enzyme reactors. Chemical Engineering Science, 31, 289-294.

KoHLwey (D. E.), CHERYAN (M.) (1981). - Performance of a $\beta$-D-galactosidase hollow fiber reactor. Enzyme Microbiology Technology, 3, 64-68.

Lewis (W.), Middleman (S.) (1974). - Conversion in a hollow fiber membrane/ enzyme reactor. AICHE Journal, 20, 1012-1014.

RoNy (P. R.) (1971). - Multiphase catalysis. Hollow fiber catalysts. Biotechnology and Bioengineering, 13, 431-447.

Silman (R. W.), Black (L. T.), Mc Ghee (J. E.), Bagley (E. B.) (1980). - Hydrolysis of raffinose in a hollow fiber reactor using an unrefined mixture of a-galactosidase and invertase. Biotechnology and Bioengineering, 22, 533-541.

WATERLAND (L. R.), Michaels (A. S.), Robertson (C. R.) (1974). - A theoretical model for enzymatic catalysis using asymetric hollow fiber membranes. AICHE Journal, 20, 1, 50-59.

Weetall (H. H.), VanN (W. P.), Pttcher (W. H.), Lee (D. D.), Lee (Y. Y.), TSaO (G. T.) (1976). - Scale-up studies on immobilized, purified glucoamylase, covalently coupled to porous ceramic support. In " Methods in enzymology, immobilized enzymes ", edited by Mosbach K., Academic Press, New York. 